\title{
Reviewer Acknowledgements for Global Journal of Health Science, Vol. 13, No. 7
}

Global Journal of Health Science wishes to acknowledge the following individuals for their assistance with peer review of manuscripts for this issue. Their help and contributions in maintaining the quality of the journal are greatly appreciated.

Global Journal of Health Science is recruiting reviewers for the journal. If you are interested in becoming a reviewer, we welcome you to join us. Please contact us for the application form at: gjhs@ccsenet.org.

\section{Reviewers for Volume 13, Number 7}

Ama Pokuaa Fenny, University of Ghana, Ghana

António Calha, Polytechnic Institute of Portalegre, Portugal

David John Lindsay, James Cook University, Australia

Diadie Maiga, World Health Organization, Regional Office for Africa, Congo

Gunta Beta, Riga Stradins University, Latvia

Hülya YARDIMCI, Ankara University, Turkey

José Joaquín Mira, Universidad Miguel Hernández, Spain

Kartheek R Balapala, University Tunku Abdul Rahman, Malaysia

Mariana de Lourdes Almeida Vieira, Centro Federal de Educação Tecnológica de Minas Gerais- CEFET, Brazil

Pedram Iranmanesh, Dentist, Independent Researcher, Iran

Pi-Ming Yeh, Missouri Western State University, United States

Samir Othman, Hawler Medical University, Iraq

Sara Melo, Queen's University Belfast, United Kingdom

Soontareeporn Meepring, Naresuan University, Thailand

Tulyakul Phatcharapon, Boromarajonani College of Nursing, Thailand 